\title{
New Anion-Exchange Resins for Improved Separations of Nuclear Materials
}

\author{
Mid-Year Progress Report \\ M ary E. Barr \\ Los Alamos N ational Laboratory
}

J une 1, 1997

\section{Goals of Project:}

We are developing multi-functional anion-exchange resins that facilitate anion uptake by carefully controlling the structure of the anion receptor site. Our new ionexchange resins interface the rapidly developing field of ion-specific chelating ligands with robust, commercial ion exchange technology. The overall objective of our research is to develop a predictive capability which allows the facile design and implementation of multi-functionalized anion exchange materials which selectively sorb metal complexes of interest from targeted process, waste, and environmental streams. The basic scientific issues addressed are actinide complex speciation along with modeling of the metal complex/functional site interactions in order to determine optimal binding-site characteristics. Our approach uses a thorough determination of the chemical species both in solution and as bound to the resin to determine the characteristics of resin active sites which can actively facilitate specific metal-complex sorption to the resin.

\section{Technical Description of W ork:}

Our technical approach combines empirical testing with theoretical modeling (applied in an iterative mode) in order to determine optimal binding-site characteristics. We determine actinide-complex speciation in specific media, then develop models for the metal complex/functional site interactions Synthesis of multi-functionalized extractants and ion-exchange materials that implement key features of the optimized binding site, and testing of these materials, provide feedback to the modeling and design activities. Resin materials which actively facilitate the uptake of actinide complexes from solution should display both improved selectivity and kinetic properties.

\section{Accomplishments to Date:}

Our first year milestones were designed to allow us to build off of our extensive expertise with plutonium in nitrate solutions prior to investigating other, less familiar systems. While the principle investigators have successfully developed actinide chelators and ion-exchange materials in the past, we were fully aware that integration of this two fields would be challenging, rewarding and, at times, highly frustrating. Relatively small differences in the substrate ( cross-linkage, impurities), the active sites (percent substitution, physical accessibility), the actinide solution ( oxidation state changes, purity) and the analytical procedures ( low detection limits) can produce inconsistent sorption behavior which is difficult to interpret. The potential paybacks for success, however,' are enormous. We feel that we have learned a great deal about how to control these numerous variables to produce consistent, reliable analysis of actinide sorption behavior with our new and baseline anion-exchange materials. The four "Year 1 (FY97)" milestones are listed below along with an update on our progress towards their completion. 
Milestone I: Complete actinide speciation analysis for actinides in nitrate solutions.

The primary actinides of interest in nitrate solutions are plutonium (IV) and americium (III). Anion exchange from nitric acid is the most frequently used process used to recover plutonium from a wide variety of residues and wastes in the DOE complex. $\mathrm{Pu}(\mathrm{IV})$ binds strongly to the anion exchange resin at high nitric acid concentrations and can be eluted with lower acid concentrations. The kinetics for sorption/desorption are extremely slow and adversely affect throughput rates. Americium is a primary contaminant in aged plutonium. Am(III), however; is less inclined to form anionic nitrate complexes in high acid media since it competes poorly with $\mathrm{H}+$. Previous extraction technology for americium has focused on cation-exchange mechanisms, but the presence of large quantities of competing species, such as Fe(III), make this process very non-selective.

Preliminary calculations based on the visible/near IR spectra of $\mathrm{Pu}(\mathrm{IV})$ at various nitric acid concentrations were the basis for our hypothesis that $\mathrm{Pu}(\mathrm{IV})$ is sorbed onto the anion-exchange site as the neutral tetranitrato complex rather than as the dianionic hexanitrato complex. This hypothesis is the basis for our search for an anion-exchange material that can actually 'facilitate' the uptake of the neutral species and it's conversion to the dianoinic complex (Figure 1).

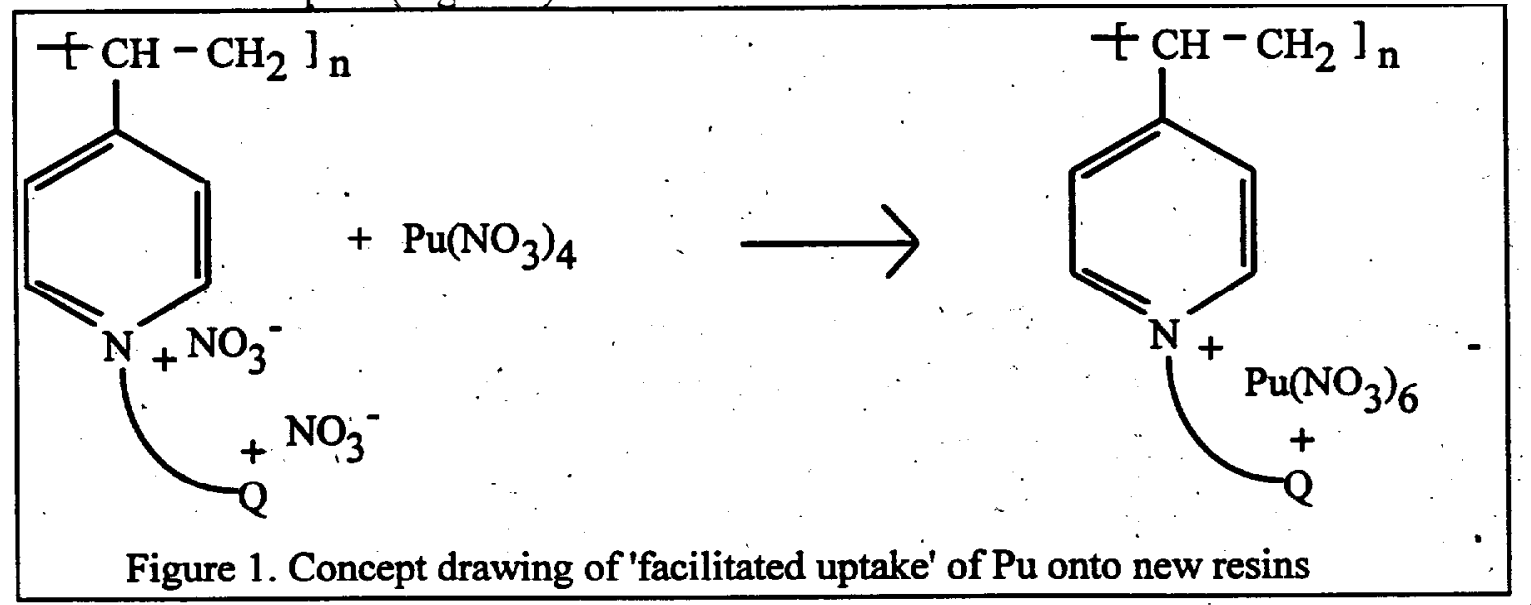

Refined formation constants for the various Pu-nitrate complexes have been determined for the mono- and di-nitrato complexes in constant ionic strength media. ( 2$20 \mathrm{~N}$ perchlorate/nitrate). Details of this experiment will soon be published in the Journal

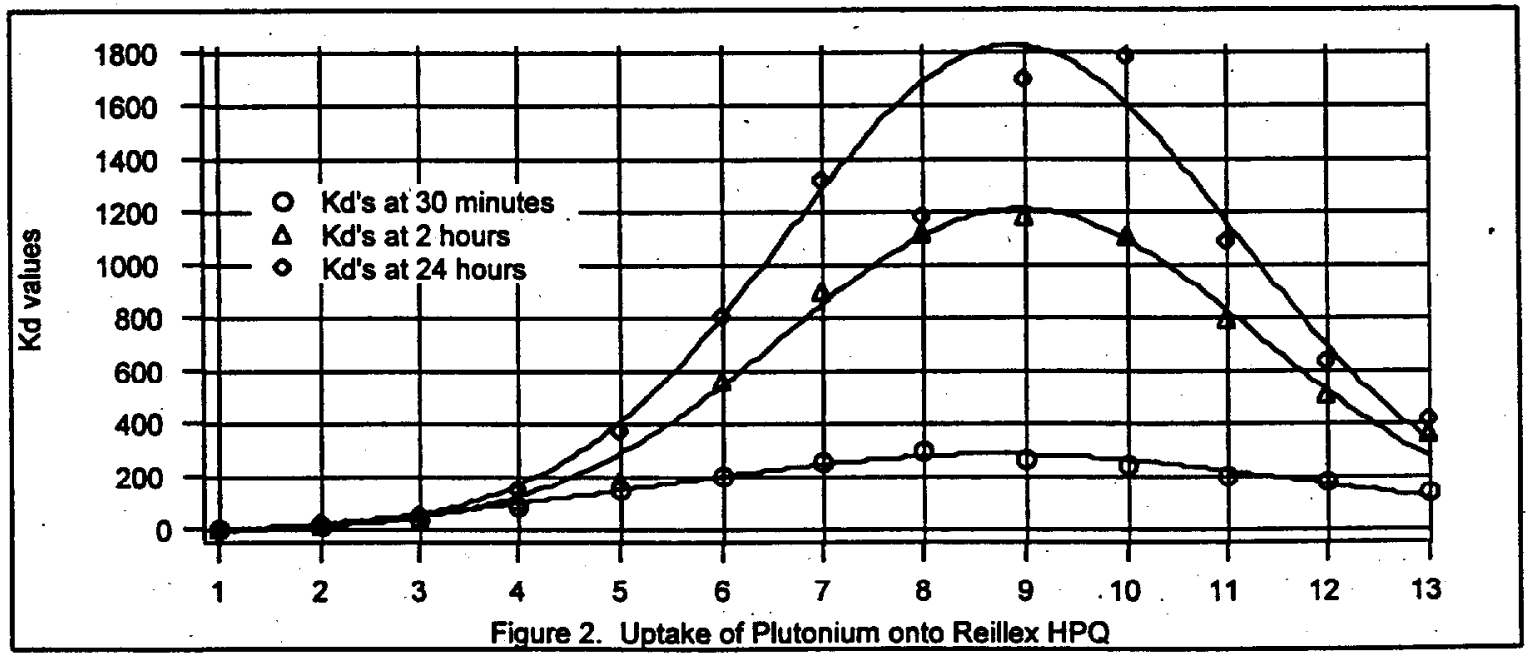


of Radioanalytical Chemistry. As expected, in higher ionic strength media, the formation of the tetranitrato complex shifts to lower acid concentrations than in pure nitric acid. We hope to use this shift to help substantiate our hypothesis of the Pu uptake mechanism.

Given the extremely slow kinetics of Pu uptake onto Reillex $\mathrm{HPQ}^{\circ}$ ( the methylated polyvinylpyridine resin currently used for plutonium processing at the Los Alamos Plutonium Facility), we could not be confident that the uptake behavior (i.e. the Kd curve) at 1/2, 2 and 6 hours corresponds to equilibrium conditions. More precise uptake experiments (Figure 2) up to 24 hours show that the uptake behavior at shorter times is reasonably indicative of equilibrium behavior. We observe 'short time' sorption and desorption behavior ( from resin loaded at $7 \mathrm{M}$ nitric acid) that is analogous to the equilibrium behavior. This helps provide support for the hypothesis that the plutonium on the anion-exchange resin is in equilibrium with the plutonium tetranitrato species in solution

Milestone 2: Complete synthesis of a systematic series of bifunctional poly(4vinylpyridine) resins, complete testing of the radionuclide sorption properties of these materials

Our 'systematic series' of bifunctional resins examines the effects of varying the following conditions for resins with the general structure shown below:

1) The chemical structure of $Q$

2) The length and chemical structure of the spacer between N and Q

3) The $\%$ crosslinking of the substrate

4) The $\%$ derivatization (i.e. ratio of bi- to mono-functional sites)

The chemical structure of 0: Our initial work was based on derivatives of Reillex 402 poly(4-vinylpyridine). This commercially available material is only lightly crosslinked (2-4\% divinylbenzene) and is relatively easy to derivatize. An example of the effects of adding the second cationic site $(\mathrm{Q})$ upon the plutonium sorption from 7 $\mathrm{M}$ nitric acid is given in Figure 3. The spacer between $\mathrm{N}$ and $\mathrm{Q}$ is a 5-

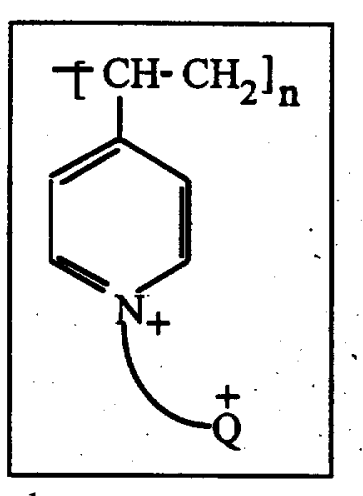
carbon pentamethylene group $\left(-\mathrm{CH}_{2} \mathrm{CH}_{2} \mathrm{CH}_{2} \mathrm{CH}_{2} \mathrm{CH}_{2}-\right.$ ). In addition to the three groups shown, we have also examined picolyl and methylated picolyl derivatives. A more comprehensive outline of the variations of $Q$ are presented in the appended manuscripts

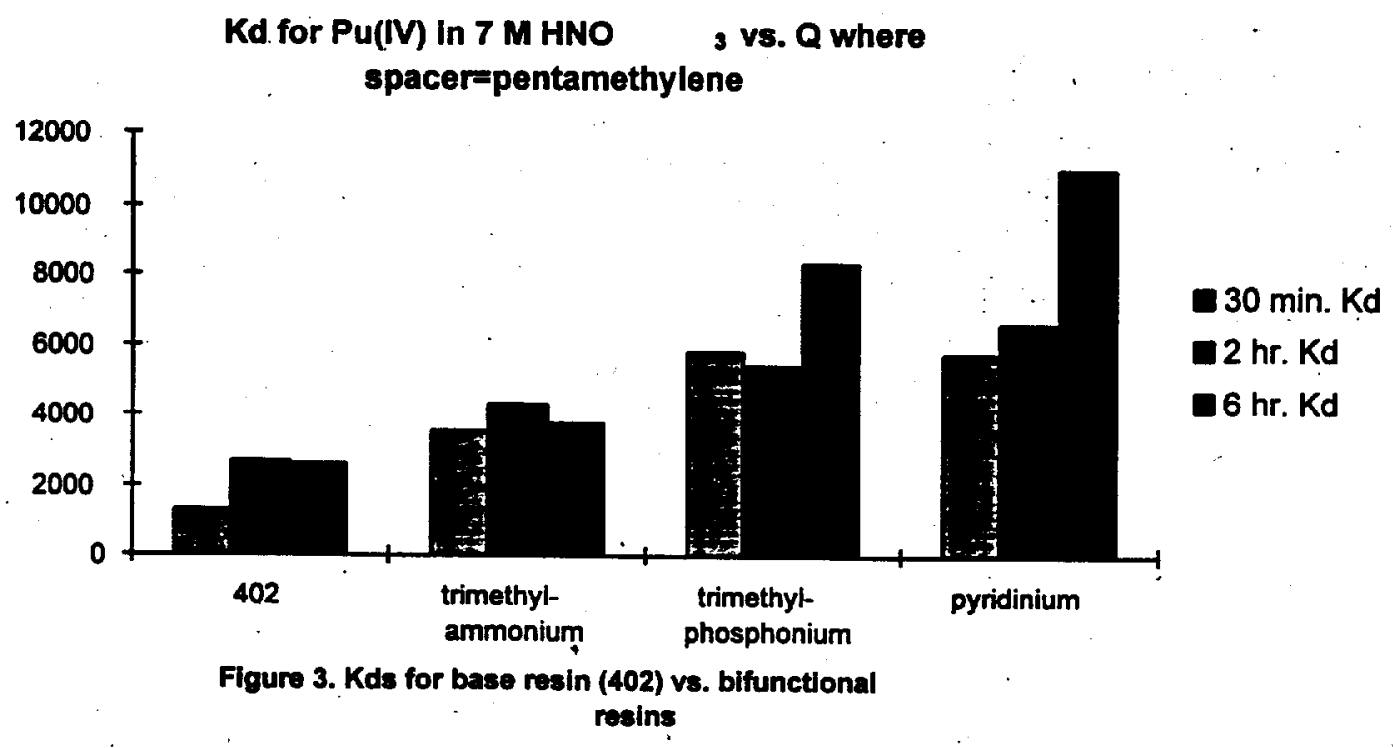


(Appendices 1 and 2).

In all cases the bifunctional resins outperformed the base polyvinylpyridine resin. For practical applications, we wish to enhance the uptake kinetics such that the resins achieve their equilibrium values very quickly. For these lightly cross-linked materials, the 30 minute Kd values are within $2 \mathrm{X}$ their 6 hour (12X longer) values. If our view of 'facilitated uptake' of Pu from nitric acid is correct, we would expect to see more rapid kinetic of Pu uptake for the bifunctional resins vs. the base resin. That we do not see such an enhancement here is somewhat disappointing, but enhanced kinetics could be 'hidden'. by the minimum contact time of 30 minutes or other complicating effects (site accessibility, hydrophilicity, etc.) which we hope to isolate in the future. As expected, variation of $\mathrm{Q}$ has relatively little impact upon the Pu uptake kinetics, but a dramatic impact upon the overall plutonium affinity. The extremely high $6 \mathrm{hr}$ (near equilibrium) $\mathrm{Kd}$ values for the bifunctional resins suggests a real thermodynamic advantage to the "preformed' di-anionic sites. The dipyridium resin exhibits the highest 6 hour Kd value.

The length and chemical structure of the spacer between $\mathrm{N}$ and Q:. An 'optimal spacer length between the two cationic sites should enhance both the plutonium uptake kinetics and thermodynamics. A single example of the impact of varied spacer length is given in Figure 4. Here again, the base resin is Reillex $402^{\circ}$ and $\mathrm{Q}$ is a second pyridinium group. Note how the 30 minute and 2 hour distribution coefficients reach a maximum value for the pentamethylene spacer. The extremely high 6 hour values for the 5- and 6- carbon spacers are less reliable, since a Kd of 10,000 means that $99.99 \%$ of the plutonium has been removed from solution and thus small uncertainties in the counting statistics result in large differences in the calculated Kd values. This general trend shows up consistently for the trimethylphosphonium and trimethylammonium derivatives. More flexible etherate linkages $\left(-\mathrm{CH}_{2} \mathrm{CH}_{2} \mathrm{OCH}_{2} \mathrm{CH}_{2}\right.$-) enhance the $\mathrm{Pu}$ affinity while more rigid spacers between $\mathrm{N}$ and $\mathrm{Q}$ result in decreased $\mathrm{Pu}$ affinity. For the picolyl derivatives, the presumption is that the orientation of the two cationic sites is sub-optimal and flexible linkages allow a more energy-minimized configuration. This issue will be addressed

\section{Distribution Coefficients vs. Spacer length}

$Q=$ pyridinium, di- to hexa-methylene spacers

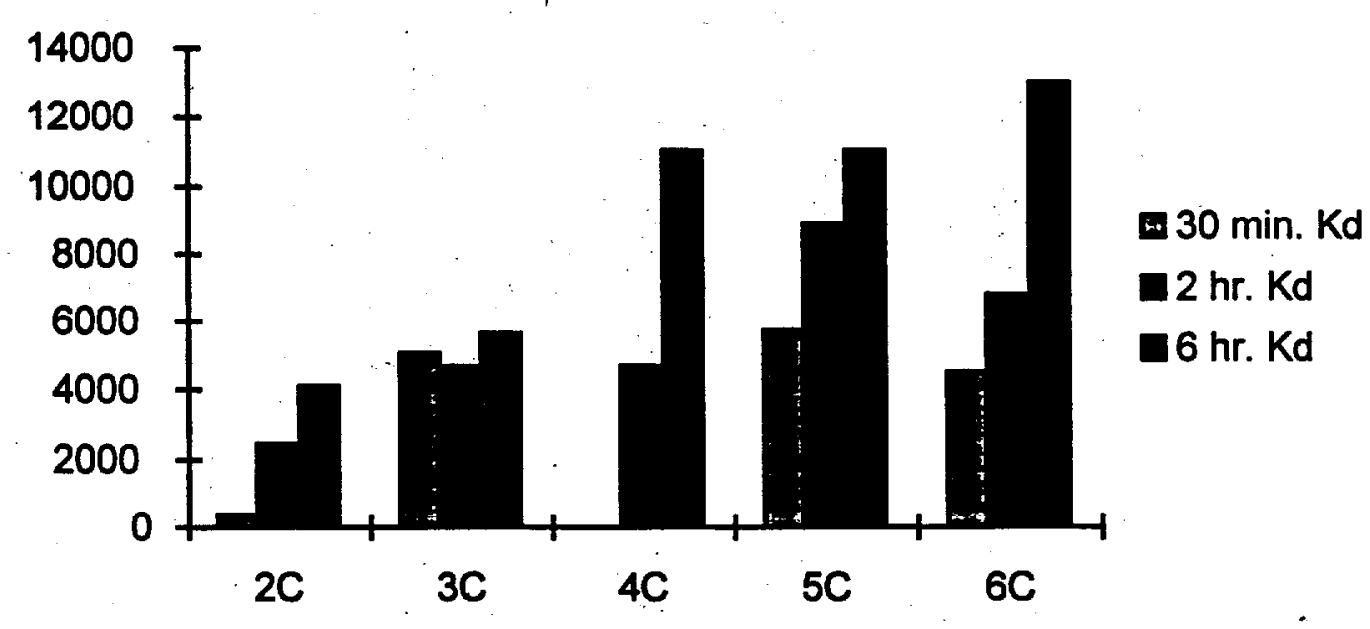

Figure 4. Kds vs. spacer length

once we have a better, handle on modeling of the cation/anion interactions. 
Unfortunately, although the 402-based resins exhibit extremely good and fast plutonium uptake behavior, the resin material is not mechanically suitable for column applications. Additionally, the 'fines' exhibit dramatically different sorption behavior (both distribution coefficients and uptake kinetics) from the 'coarse' fractions which produces erratic results. More highly cross-linked resins are required in order to maintain the physical rigidity necessary for column applications.

Effect of resin crosslinking and percent derivatization: Very low cross-linked polyvinylpyridine is quite permeable and hydrophilic. Thus, anions in solution have ready access to the weak-base cationic sites. Increased crosslinking (via divinylbenzene) decreases hydrophilicity and permeability, but increases physical integrity. Reilly Industries kindly provided us with non-commercial samples of $18 \%$ and $21 \%$ crosslinked macroporous poly(4-vinylpyridine). These materials, along with the commercially available Reillex $\mathrm{HP}^{\oplus}$ (approximately $25 \%$ crosslinked), were derivatized to form bifunctional resins $\left(-\mathrm{CH}_{2} \mathrm{CH}_{2} \mathrm{CH}_{2} \mathrm{CH}_{2} \mathrm{CH}_{2} \mathrm{~N}^{+} \mathrm{Me}_{3}\right)$ and monofunctional resins (-Me). Methylation of the polyvinylpyrdine base resin converts single weak-base sites to single strong-base sites, while bifunctionalization converts each weak-base site to essentially two strong-base sites. As seen in Figure 5, the bifunctional resins outperform the monofunctional resins of the same crosslinking percentage. Although we have not derivatized the bifunctional materials to near $100 \%$, it appears that they continue to perform better at higher percent derivatization rather than leveling out as do the monofunctional resins.

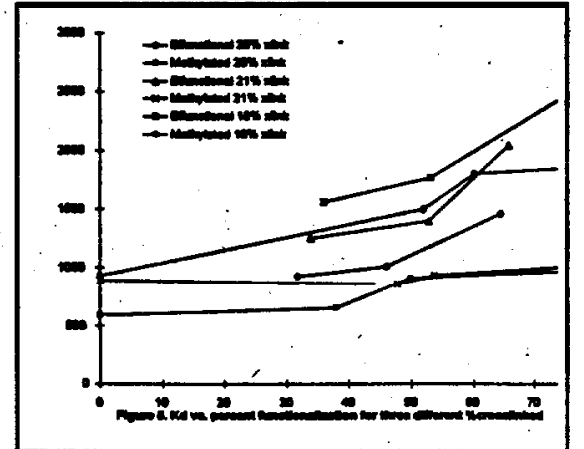

Milestone 3: Synthesize a partial set of the multifunctional extractants for structural and speciation studies.

Relatively little work has been completed on this milestone to date (June 1). We have synthesized several bipyridinium ligands (both hydrophilic and lipophilic) with two and six methylene spacers. Over the remaining four months, we will conduct biphasic extraction studies and spectroscopic studies of the effect of these ligands upon the Pu and Am equilibrium complexation in nitric acid. Some analysis of the thermodynamics (primarily heat of formation) of complexation should also be useful in supporting the modeling studies.

Milestone 4: Develop molecular modeling parameters for relevant actinide complexes.

We have made considerable progress in describing actinide complexes in nitrate media (Figure 6). Although relatively little. structural information is available for Pu and Am compounds, we have been able to combine that information with structural data for uranium and lanthanide nitrato complexes to refine the actinide MM2 parameters. This has been accomplished by performing ROHF (Hartree-Fock) calculations to achieve realistic partial charges on the metal, coordination sphere, and the complexed ligand for $\mathrm{Pu}\left(\mathrm{NO}_{3}\right)_{\mathbf{x}^{\mathrm{q}}}{ }^{-}$and pyridine/spacer/quaternary ammonium salt ligands. Once these charges were computed, the problem of accurately determining the force constants was undertaken. The parameters were adjusted to fit the experimental EXAFS and x-ray data with the following goals: One, the $\mathrm{Pu}-\mathrm{O}$ bonds were set to the lowest possible value to give the model the maximum amount of flexibility. Two, the O-N-O angle bending parameter was set to the lowest possible value again to give the model the maximum amount of flexibility. Three, the NO3 group was assigned a out-of-plane bending constant of $99 \mathrm{mdyn} / \AA$ so that the nitrate group would remain planar. The out-of-plane 
$\mathrm{NO}_{3}$ approach accelerates calculations since fewer -calculations are required at each step. In several of our models, it has been noted that the nitrate group would pivot along the $\mathrm{O}_{2}-\mathrm{Pu}-\mathrm{O}_{2}$ plane to better dock with the ligand, this is very encouraging since the same effect is seen in some EXAFS work, and the model is able to predict this rotation without any parameters to describe this effect. Presently; a QSAR (quantum structural activity relationships) study of the coloumbic charges and steric energy versus $K_{d}$ values is being undertaken for different functional units in the ligand, different spacers, and different solvent effects (i.e., in 9M nitric acid versus $3 \mathrm{M}$ nitric acid).

We plan the following: One, to refine the parameters to 'accelerate convergence; at the moment it takes between 1,000 and 2,000 iterations to converge a model. Two, to

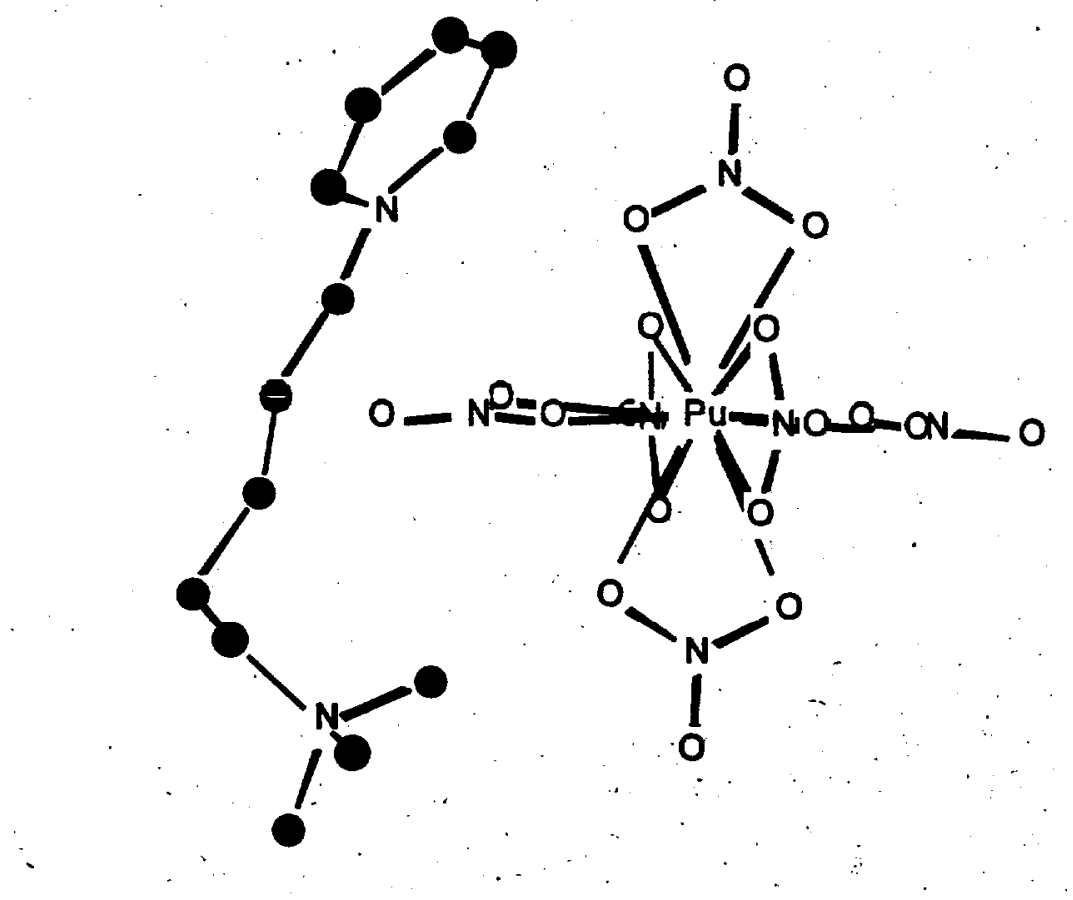

Figure 6. A view of $\mathrm{C}_{5} \mathrm{H}_{5} \mathrm{~N}\left(\mathrm{CH}_{2}\right)_{5} \mathrm{~N}(\mathrm{Me})_{3}$ with $\mathrm{Pu}\left(\mathrm{NO}_{3}\right)_{6}{ }^{3+}$ GAMESS. translate the above parameters to HyperChem, since HyperChem is a faster program. Three, a more extensive quantum mechanical treatment of the ligand systems using MCSCF (selfconsistent field theory): Four, extend the parameter set to include the effects of solvation (in different acid concentrations). We plan on. performing the above. tasks with HyperChem, CSC Chem3D+, and the ab initio program 


\section{Projections:}

We anticipate near-completion of the FY97 scope by the end of September. There appears to be considerable confusion over whether the 'year' runs Sept. 1-Sept. 1 as per the original proposal or Oct. 1-Oct. 1 as per funding. My current assumption is the normal Oct.-Oct. FY (it's easier that way). Our university affiliate, RA. Bartsch of Texas Tech University, has recently received funding for his part of the project for a May-May year. This delay in funding ( 9 months) had a negative impact upon his ability to synthesize the anion-exchange materials and the corresponding extractants since he was unable to purchase the required high-pressure reaction vessel. We were able to mitigate the adverse effect by reshuffling our schedule and modifying some of our synthetic goals. If all goes well, we should be able to achieve most of our FY goals in the remaining months.

Much of the remaining work is outlined under the individual milestones listed above. We still have some americium speciation work remaining, and collected EXAFS data for $\mathrm{Pu}$ on the anion-exchange materials still needs to be processed and analyzed. We want to synthesize and evaluate bifunctional resins with higher percent derivatization ( we have up to $70 \%$ ), and the existing data needs to be more comprehensively collated and correlated. We plan on testing extractants and ligands which represent the 'extremes' of behavior observed for the bifunctional resins (those which exhibit good/bad $\mathrm{Pu}$ and $\mathrm{Am}$ uptake). Molecular modeling of the covalent and electrostatic interactions in high ionic strength media is ongoing.

\section{F unding:}

Due to the delayed funding for our academic collaborator, we deferred certain costs in case we needed to supply funds to cover his work. Now that he has received his allocations, those bills need to be paid. To date, we have received $298 \mathrm{~K}$ in allocations. To date, we have spent $164 \mathrm{~K}$ and project another $150 \mathrm{~K}$ in expenditures for the remaining months. This total amount is in slight excess of current allocations, but in line' with the original funding projections. There still seems to be a $20 \mathrm{~K}$ discrepancy between the funding level authorized (1.3 M for three years with \$318 in year 1 for LANL) and the received allocations (see the April progress report for full details).

\section{I ssues/Problem:}

The delay in receiving allocations both at LANL and at TTU has really been more of an irritation and a test of our ingenuity than a true show-stopper. We managed to conduct some work outlined in the out-year goals here at LANL when TTU was not able to supply new materials. Therefore, there has been some 'mixing' of the milestones. I don't see that as a major problem, though. Milestones for basic research projects are necessarily fuzzy, since new knowledge inevitably impacts old knowledge. Come October I, we won't turn our back on the FY97 work, but rather will integrate the new information gained on other media with what we have learned. The fundamental strength of multi-year support for basic research, is that it allows us to continually build on an ever-expanding foundation, such that each year's efforts are more productive. Thus, I believe that any deficiencies in our 97 studies can easily be made up in 98/99.

\section{Corrective Actions: \\ See the I ssues/Problem above.}




\section{Additional Information:}

Research conducted under the aspecies of this project has been presented at the' following national/international meetings and published in the following journals:

"Development of Anion-Exchange Resins for Separations of Actinides", talk presented at the "Ligand Design for Ion Separations", I\&EC division, 213th ACS National Meeting: BARR ME, JARVINEN GD, MARSH SF, BARTSCH RA, ABSTRACTS OF PAPERS OF THE AMERICAN CHEMICAL SOCIETY, v. 213(pt.2) pp. 73-IEC 1997.

"Bifunctional Anion-Exchange Resins for Improved Separations of Nuclear Materials" talk presented at the MARC IV International Conference of Methods and Applications of Radioanalytical Chemistry, April 1997, Barr ME, Jarvinen GD, Marsh SF, Bartsch RA

"New Bifunctional Anion-Exchanged Resins for Nuclear Waste Treatment" Barr ME, Jarvinen GD, Marsh SF, Bartsch RA, Reactive and Functional Polymers, in press.

'New Bifunctional Anion-Exchange Resins for Nuclear Waste Treatment- Part II" Barr ME, Jarvinen GD, Marsh SF, Bartsch RA, submitted to Journal of Radioanalytical and Nuclear Chemistry. 